\title{
Self-healing of hyaluronic acid to improve in vivo retention and function
}

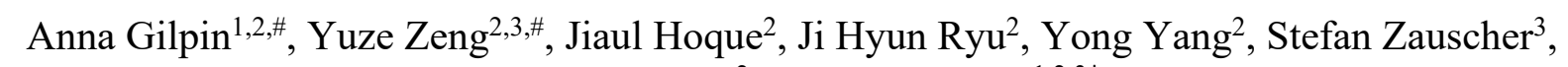
William Eward ${ }^{2}$, Shyni Varghese ${ }^{1,2,3^{*}}$

${ }^{1}$ Department of Biomedical Engineering, Pratt School of Engineering, Duke University, Durham, NC 27710

${ }^{2}$ Department of Orthopaedic Surgery, Duke University School of Medicine, Duke University, Durham, NC 27710

${ }^{3}$ Department of Mechanical Engineering and Materials Science, Duke University, Durham, NC 27710

${ }^{\#}$ These authors contributed equally to this work

*To whom Correspondence should be addressed

Email: shyni.varghese@duke.edu; Tel: +1-919-660-5273

Keywords: lubrication; self-healing; cartilage; anterior cruciate ligament transection; osteoarthritis 


\section{Abstract}

2 Convergent advances in the field of soft matter, macromolecular chemistry, and engineering have

3 led to the development of biomaterials that possess autonomous, adaptive, and self-healing

4 characteristics similar to living systems. These rationally designed biomaterials could surpass the

5 capabilities of their parent material. Herein, we describe the modification of hyaluronic acid (HA)

6 molecules to exhibit self-healing properties and studied its physical and biological function both

7 in vitro and in vivo. Our in vitro findings showed that self-healing HA designed to undergo

8 autonomous repair improved lubrication, enhanced free radical scavenging, and resisted enzymatic

9 degradation compared to unmodified HA. Longitudinal imaging following intra-articular injection

10 of self-healing HA showed improved in vivo retention despite the low molecular weight.

11 Concomitant with these functions, intra-articular injection of self-healing HA mitigated anterior

12 cruciate ligament injury-mediated cartilage degeneration in rodents. This proof-of-concept study

13 shows how incorporation of functional properties like self-healing can be used to surpass the 14 existing capabilities of biolubricants. 


\section{Introduction}

19 Incorporating distinct molecular and chemical features into biomaterials can introduce new

20 functionalities to combat intrinsic limitations of the parent materials towards their desired

21 application. For example, biomaterials that can undergo in situ self-repair could not only improve

22 their in vivo longevity and function but could also promote their therapeutic outcome. Herein, we

23 examine whether hyaluronic acid molecules designed to undergo self-healing could improve their

24 physical and biological functions with a focus on biolubrication. Biolubrication is critical for the

25 efficient function of diarthrodial joints, eyes, lungs and other visceral organs. The lubricating

26 molecules present at the articular cartilage interfaces of the diarthrodial joints maintain tissue

27 health and tribological functions. ${ }^{[1]}$ Enabling inter-surface lubrication is also important for the

28 seamless function of various medical devices. Due to its prevalence in biolubrication and

29 biological functions ${ }^{[2]}$ and its alteration in diseases like osteoarthritis, ${ }^{[3]} \mathrm{HA}$ has been widely used

30 to treat lubrication deficiencies. ${ }^{[4]}$ Besides promoting lubrication, HA also exhibits various

31 biological functions, such as reducing inflammation and free radical damage, as well as alleviating

32 pain. ${ }^{[5]}$ However, a persistent challenge to HA lubricants is their short in vivo residence time. ${ }^{[6]}$

33 Hence, strategies that ensure long-term retention and function of exogenous HA have been

34 developed to improve its clinical outcome. ${ }^{[7]}$ One of the most widely used approaches to enhance

35 the in vivo longevity of HA includes the introduction of chemical crosslinks; however, this strategy

36 severely limits HA's lubricating function and its handling. ${ }^{[5 a, 8]}$ Albeit at low incidence, chemical

37 crosslinking can also result in pseudoseptic reactions. ${ }^{[9]}$

39 We introduced self-healing and dynamic crosslinking into HA molecules and assessed their

40 potential to improve the physical and biological functions of parent HA without compromising its 
41 injectability. Towards this, we modified HA with ureidopyrimidinone (UPy) groups to enable self-

42 healing of HA polymers under physiological conditions, via reversible secondary interactions. ${ }^{[10]}$

43 Biomaterials modified with UPy molecules have been exploited for various biomedical

44 applications. ${ }^{[11]}$ UPy molecules rapidly dimerize through quadruple hydrogen bonding resulting in

45 dynamic supramolecular structures under physiological conditions. ${ }^{[10,12]} \mathrm{HA}$ molecules endowed

46 with UPy moieties can form a dynamic network through hydrogen bonding while exhibiting shear-

47 thinning behavior (via reorganization of the polymer chains in response to shear forces), thus

48 enabling easy injection and efficient lubrication. At rest, the rapid UPy dimerization re-establishes

49 the stable supramolecular network, and these "self-generating" networks will resist rapid clearance

50 from the synovial space (Fig. 1a). Thus, the self-healing HA molecules will offer the benefits of

51 both high molecular weight HA (shear thinning, mechanical adaptability, and enhanced

52 lubrication), as well as chemically crosslinked HA (improved in vivo retention and reduced

53 enzymatic degradation). As a proof-of-concept, we modified $200 \mathrm{kDa}$ HA molecules with UPy

54 moieties and assessed whether self-healing capability could improve the in vivo retention and

55 chondroprotective function of HA in articular cartilage lubrication. We chose to use low molecular

56 weight HA because it lacks viscoelasticity and other beneficial effects towards in vivo retention,

57 thus eliminating any confounding contributions.

59 Results and discussion

\section{Synthesis and characterization of HA-UPy molecules}

61 HA-UPy molecules were prepared by functionalizing HA with UPy-bearing linkers as shown in

62 the reaction scheme (Fig. 1b). Details of the UPy linker synthesis are provided in Methods and 


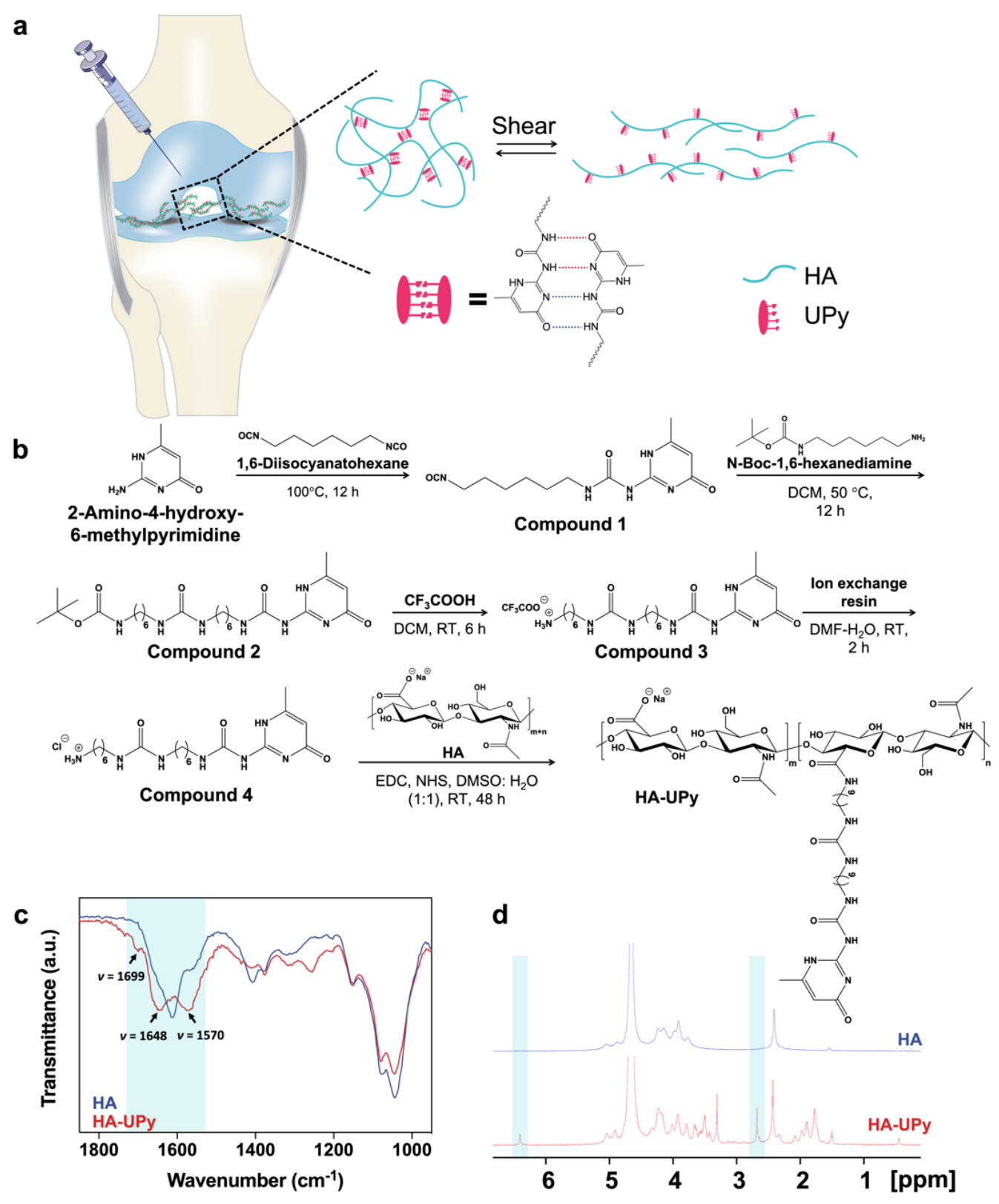

Figure 1. Development of self-healing HA lubricant (i.e, HA-UPy). $\boldsymbol{a}$, Schematics of intraarticular injection of self-healing HA lubricant. When under shear during injection or joint movements, HA-UPy becomes fluidic and spreads in the joint, lubricating the articular surface. After the removal of shear force, HA-UPy can self-generate into a network via UPy-mediated hydrogen bonding, contributing to its retention in the joint. b, Synthesis route. DCM: dichloromethane; DMF: dimethylformamide; DMSO: dimethyl sulfoxide; EDC: 1-ethyl-3-(3dimethylaminopropyl) carbodiimide hydrochloride; NHS: N-hydroxysuccinimide; RT: room temperature. $\boldsymbol{c}$, FTIR spectra of HA and HA-UPy. $\boldsymbol{d}$, ${ }^{1} \mathrm{H}$ NMR spectra. Pyrimidinone protons of UPy: $\delta 6.41$ ppm $(=\mathrm{CH}-)$ and $\delta 2.69 \mathrm{ppm}\left(-\mathrm{CH}_{3}\right)$. 
64 Supplementary Information (Supplementary Figs. 1-5 and Supplementary Note). The linker

65 was designed to bear the UPy moiety on one end ${ }^{[12 \mathrm{a}]}$ with the other end containing a primary amine

66 group protected by a cleavable tert-butyloxycarbonyl (Boc) group. Upon removal of the Boc

67 group, the primary amine on the linker reacts with the carboxylic acid of the HA's D-glucuronic

68 acid, resulting in UPy conjugation to HA. The Fourier-transform infrared (FTIR) spectroscopy of

69 the product confirmed UPy conjugation with new peaks appearing at 1699, 1648, and $1570 \mathrm{~cm}^{-1}$,

70 corresponding to pyrimidinone $\mathrm{C}=\mathrm{O}$ stretching, urea $\mathrm{C}=\mathrm{O}$ stretching, and pyrimidinone $\mathrm{C}=\mathrm{N}$

71 stretching, respectively (Fig. 1c). The UPy conjugation was further confirmed by proton nuclear

72 magnetic resonance ( $\left.{ }^{1} \mathrm{HNMR}\right)$ spectroscopy (Fig. 1d). We estimated a grafting density of $\sim 24 \pm$

$733 \%$ per dimeric repeating unit of HA from the ${ }^{1}$ HNMR spectra by comparing the integrated peak

74 area of the pyrimidinone protons in a UPy unit $\left(-\mathrm{NH}-\mathrm{C}\left(\mathrm{CH}_{3}\right)-\mathrm{C} \underline{\mathrm{H}}-\mathrm{CO}-, 1 \mathrm{H}, \delta 6.41\right.$;

$\left.75-\mathrm{NH}-\mathrm{C}\left(\underline{\mathrm{CH}_{3}}\right)-\mathrm{CH}-\mathrm{CO}-, 3 \mathrm{H}, \delta 2.69\right)$ to that of the acetyl protons in HA's N-acetyl-D-

76 glucosamine unit (-NH-CO- $\underline{\mathrm{CH}_{3}}, 3 \mathrm{H}, \delta$ 2.45) (Fig. 1d and Supplementary Fig. 6). For live in

77 vivo imaging (IVIS), HA and HA-UPy molecules were conjugated with the near infrared (NIR)

78 fluorescent dye cyanine 7 (Cy7) (Supplementary Fig. 7).

\section{HA-UPy molecules form supramolecular networks and exhibit self-healing}

81 The UPy-mediated non-covalent interactions among the polymer chains can facilitate self-

82 assembly of HA molecules into dynamic networks (i.e., soft gels), which we characterized by

83 rheological measurements. To study the effect of polymer concentration, we prepared solutions of

84 HA-UPy and HA at three concentrations (2, 5, and $10 \mathrm{wt} \%)$ in phosphate-buffered saline (PBS).

85 The frequency sweep $(0.1-10 \mathrm{~Hz})$ measurements of HA-UPy and unmodified HA showed that

86 HA-UPy samples exhibit higher G' (storage modulus) and G" (loss modulus) at all frequencies 

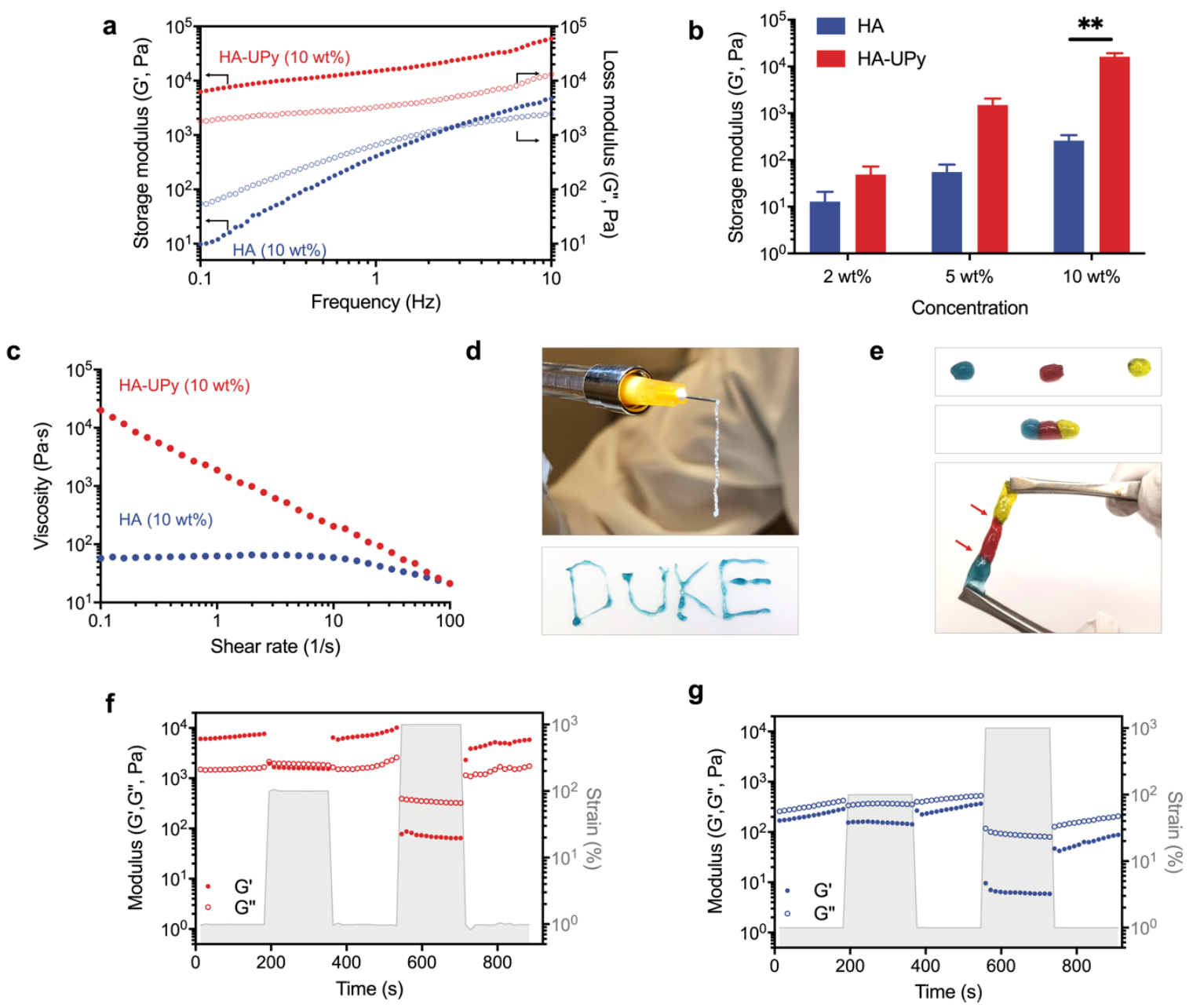

Figure 2. Characterization of self-healing HA. $\boldsymbol{a}$, Storage $\left(\mathrm{G}^{\prime}\right)$ and loss $\left(\mathrm{G}^{\prime \prime}\right)$ moduli of HAUPy and HA in a frequency sweep measurement. $\boldsymbol{b}$, Storage modulus $\left(\mathrm{G}^{\prime}\right)$ of HA-UPy and HA as a function of concentration. $c$, Viscosity changes of HA-UPy and HA as a function of shear rate. $\boldsymbol{d}$, HA-UPy $(10 \mathrm{wt} \%)$ was easily extruded through a $26 \mathrm{G}$ needle into "DUKE" letters. $\boldsymbol{e}$, Separate pieces of HA-UPy $(10 \mathrm{wt} \%)$ hydrogels healed together with interfaces indicated with red arrows. $f, g$, Step-strain measurements for $10 \mathrm{wt} \% \mathrm{HA}-\mathrm{UPy}(\mathbf{f})$ and $10 \mathrm{wt} \% \mathrm{HA}(\mathbf{g})$.

while the HA samples behaved more like a viscous liquid ${ }^{[13]}$ (Fig. 2a and Supplementary Figs.

8a, b). Furthermore, the storage modulus, determined at $1 \mathrm{~Hz}$, of the HA-UPy samples increased with increasing concentration (Fig. 2b). The oscillation frequency of $1 \mathrm{~Hz}$ was chosen because it is within the range of typical walking frequencies. ${ }^{[14]}$ The concentration-dependent network 
91 formation was also visualized by inverting the tubes containing the polymer solutions. The samples

92 containing HA-UPy showed solid-like behavior at higher concentrations and did not flow like a

93 liquid. In contrast, the samples containing unmodified HA behaved like a viscous liquid at all

94 concentrations, with the $10 \mathrm{wt} \%$ solution taking a longer time to flow (Supplementary Fig. 8c).

95 These observations for the HA-UPy samples are consistent with network formation, which arises

96 from quadruple hydrogen bonding between the UPy moieties. Further experiments were carried

97 out using $10 \mathrm{wt} \%$ HA and HA-UPy.

99 Because the UPy-mediated network formation is dynamic, the HA-UPy samples should show shear-thinning and self-healing functions. As expected, the viscosity of the HA-UPy samples

101 decreased with increasing shear rate, showing a characteristic shear-thinning behavior which 102 results from the destruction of the physical crosslinks by the applied shear stresses (Fig. 2c). In 103 contrast, the shear rate-dependent viscosity of the corresponding HA solution is consistent with 104 that of a viscous liquid. The HA-UPy samples (10 $\mathrm{wt} \%)$ were easily injected, with minimal 105 resistance, through a 26G hypodermic needle (Fig. 2d). The extruded HA-UPy formed a stable 106 network at rest, which enabled the "printing" of different shapes (Fig. 2d).

108 We examined the self-healing of HA-UPy, following an approach reported previously, ${ }^{[15]}$ by 109 bringing multiple pieces of HA-UPy hydrogels into close contact, which showed instantaneous 110 healing (Fig. 2e). Furthermore, we used step-strain measurements to confirm the UPy-mediated 111 self-healing of HA-UPy and dynamic networks, wherein $10 \mathrm{wt} \%$ HA-UPy and HA samples were 112 subjected to alternating step strains of 1 to $100 \%$ and 1 to $1000 \%$ (Figs. 2f, g). The storage modulus $113\left(G^{\prime}\right)$ values of the HA-UPy samples dropped to that of the loss modulus ( $\left.G^{\prime \prime}\right)$ at a strain $\gamma=100 \%$, 
114 indicating network disruption (Fig. 2f). When the strain was removed, the HA-UPy molecules re-

115 organized and formed a new network structure instantaneously with a $100 \%$ recovery of G'.

116 Increasing the strain rate to $1000 \%$ induced more network destruction as indicated by the drop of

117 the storage modulus to $\sim 100 \mathrm{~Pa}$, with a corresponding inversion of $\mathrm{G}^{\prime}$ and $\mathrm{G}^{\prime \prime}$, suggesting liquid-

118 like flow behavior. Despite the large strain $(\gamma=1000 \%)$, prompt recovery of the network structure

119 was observed upon the removal of the strain. Unmodified HA samples, on the other hand, had a

120 higher loss modulus than storage modulus at both low and high strains (Fig. 2g). This behavior is

121 indicative of a viscous liquid. Additionally, we performed experiments where we alternatingly

122 applied a low (1\%) and high (500\%) strain over multiple cycles to determine whether the HA-UPy

123 sample would recover its storage modulus after repeated network disruptions. These studies

124 showed complete network formation without hysteresis as indicated by the $\mathrm{G}^{\prime}$, which maintained

125 its original value at $1 \%$ strain following repeated network disruption at $\gamma=500 \%$ (Supplementary

126 Fig. 9).

127

128 Self-healing HA exhibits enhanced lubrication

129 Effectiveness of the biolubricant to reduce friction between the articular surfaces is key to its

130 application in improving joint function. We thus investigated whether UPy-mediated changes in

131 the viscoelastic properties of HA-UPy would translate to its lubrication function. To this end, we

132 determined the coefficient of friction $(\mu)$ between healthy porcine cartilage explants in the presence

133 of HA-UPy and compared the measured values with those obtained when using corresponding HA

134 solutions and saline (negative control) by using a rotational rheometer. We determined the

135 coefficient of friction (COF), $\mu$, at the cartilage-to-cartilage interface, using the equation, $\mu=$

$136 \frac{\text { Shear stress }}{\text { Normal stress }}$. Figure 3a shows that the COF between the contacting articular surfaces decreased 
137 significantly in the presence of HA-UPy. Specifically, the HA-UPy molecules reduced friction by

$138 \sim 70 \%$ and $55 \%$ compared to saline and HA molecules, respectively.

140 Self-healing HA promotes free radical scavenging

141 HA has a number of biological functions, including serving as an antioxidant to reduce free radical

142 damage to cells. We investigated the free radical scavenging effect of HA-UPy using 143 deoxyribose/Fenton reagent and 1,1-diphenyl-2-picrylhydrazyl (DPPH) assays. ${ }^{[16]}$ In the

144 deoxyribose/Fenton reagent assay, hydroxyl radicals are produced by the reaction of $\mathrm{Fe}^{2+}-\mathrm{EDTA}^{2}$ 145 with hydrogen peroxide. The hydroxyl radicals subsequently interact with deoxyribose and form 146 a pink color chromogen with thiobarbituric acid upon heating. Following incubation with the 147 Fenton reagent, the absorbance of the solution was measured. As seen in Figure 3b, the solution 148 incubated with HA-UPy had a lower chromogen absorbance, corresponding to fewer hydroxyl 149 radicals, suggesting free radical scavenging by HA-UPy molecules. Since free radicals cleave the 150 glycosidic bonds in HA which could lead to the breakdown of polymer chains, we examined the 151 storage moduli of the HA-UPy exposed to the Fenton reagent and compared them to the storage 152 moduli of untreated HA-UPy samples. Figure 3c shows a slight reduction in the $\mathrm{G}^{\prime}$ value, 153 indicating some disruption of the network in the presence of free radicals (Supplementary Fig. 154 10). Although we stopped the reaction after $1 \mathrm{~h}$, ensuring the complete removal of free radicals 155 from the solution is challenging. It is thus likely that free radicals continued to react with HA-UPy 156 molecules. While the Fenton assay enables us to examine the free radical scavenging effect of the 157 HA-UPy in a physiologically relevant environment, a potential radical scavenging property of 158 unmodified HA molecules cannot be determined because of the aqueous reaction environment. 

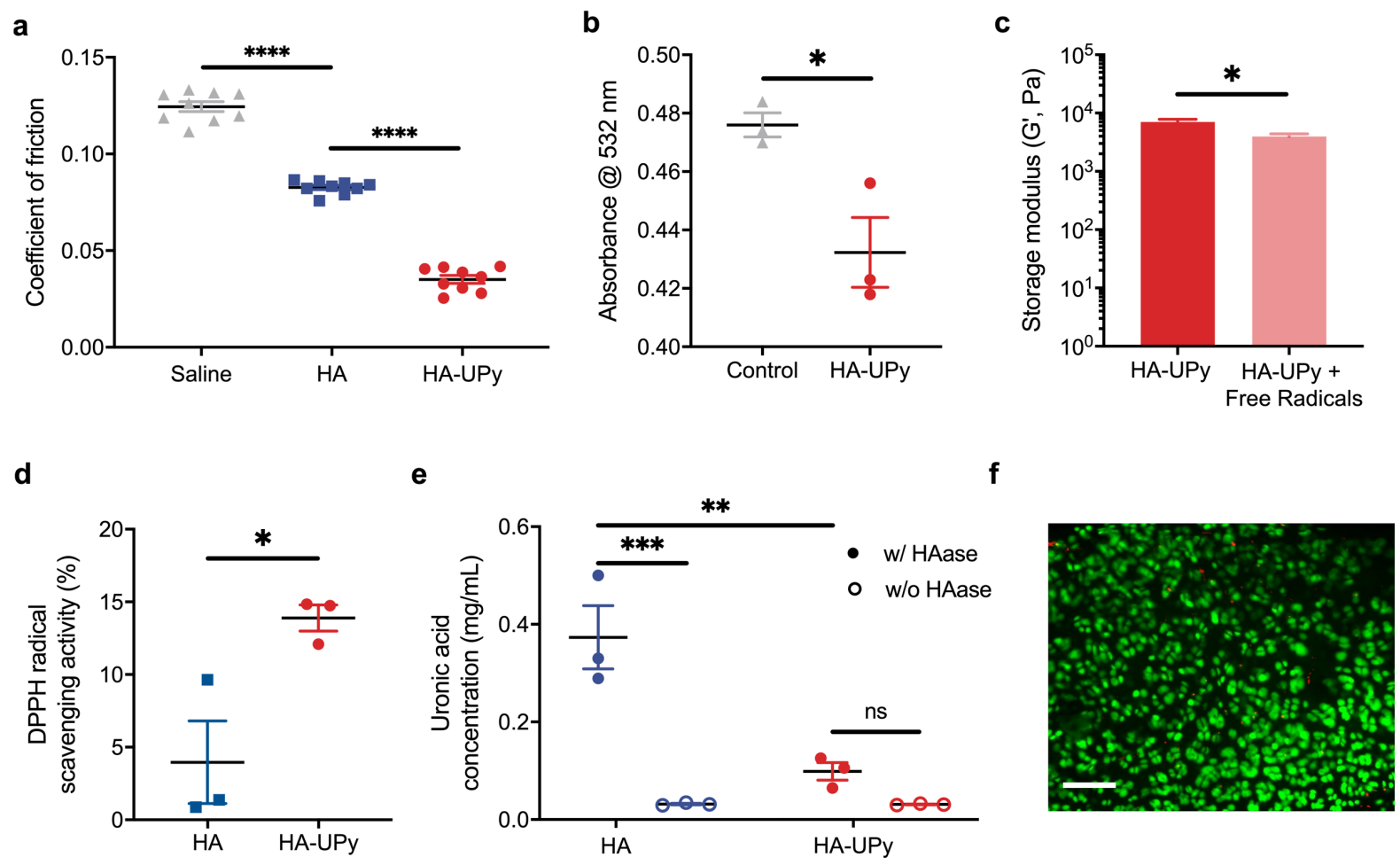

Figure 3. Lubrication, antiradical capacity, in vitro stability, and cytocompatibilty of selfhealing HA. $\boldsymbol{a}$, Coefficients of friction at the cartilage-cartilage interface in the presence of HAUPy, HA, and saline. $\boldsymbol{b}$, Free radical scavenging effect of HA-UPy exposed to Fenton reagent compared to the phosphate buffer control. $c$, Storage modulus $\left(\mathrm{G}^{\prime}\right)$ of HA-UPy measured at a frequency of $1 \mathrm{~Hz}$ following exposure to hydroxyl radicals compared to non-exposed HA-UPy. $\boldsymbol{d}$, DPPH radical scavenging of HA-UPy compared to HA. $\boldsymbol{e}$, Degradation products of HA-UPy and HA with and without the presence of hyaluronidase (HAase). $f$, Chondrocyte viability in a rat cartilage explant after $7 \mathrm{~d}$ incubation with HA-UPy. Green: live cells; Red: dead cells. Scale bar: $100 \mu \mathrm{m}$. All data are presented as means ( \pm s.e.m.). One-way ANOVA (a), unpaired twotailed t-test $(\boldsymbol{b}, \boldsymbol{c}, \boldsymbol{d})$ or two-way ANOVA $(\boldsymbol{e})$ with Tukey's multiple-comparisons test was used for statistical analysis. Significance is determined as $* P<0.05, * * P<0.01, * * * P<0.001$, $* * * * P<0.0001$ and n.s. (not significant).

Hence, we also used a DPPH assay to examine the UPy-mediated changes in free radical

161 scavenging, where the samples were exposed to a DPPH solution in ethanol. The solution

162 containing HA-UPy molecules showed a significantly reduced DPPH free radical concentration as

163 compared to that containing HA, which had a minimal scavenging effect (Fig. 3d). The free 
radical scavenging ability of HA-UPy could be due to the network formation and/or the presence of UPy moieties. Prior studies have showed that the protective effect of HA against the free radical damage to the cells depends on HA molecular weight, with high molecular weight HA providing

167 better protection. ${ }^{[17]}$ Furthermore, UPy moieties contain pyrimidine rings, which are known to 168 scavenge free radicals. ${ }^{[18]}$ The minimal reduction in $\mathrm{G}^{\prime}$ of HA-UPy following free radical exposure 169 could be attributed to the UPy-mediated self-healing/self-generation of networks or by the UPy scavenging the free radical itself.

\section{Self-healing HA molecules resist enzymatic degradation}

173 HA within the synovial fluid is subjected to enzymatic and free radical degradation as well as

174 lymphatic drainage, which are some of the key players contributing to its rapid clearance in the 175 joint. The short residence time $\left(\mathrm{t}_{1 / 2} \sim 24 \mathrm{~h}\right)$ of HA within the synovial joint ${ }^{[19]}$ has been thought to

176 be one of the factors contributing to its limited clinical effectiveness following intraarticular 177 injection, and chemically crosslinked HA derivatives have thus been generated to delay or slow 178 the breakdown. ${ }^{[5 a, 20]}$ We posit that the formation of supramolecular HA networks by UPy 179 interactions may also slow the degradation of HA molecules. To test this, we incubated HA-UPy with hyaluronidase and quantified the resultant HA fragments by using a modified uronic acid 181 assay. ${ }^{[21]}$ As demonstrated by the results, the HA-UPy experienced minimal degradation compared 182 to the corresponding HA in the presence of hyaluronidase. Moreover, no statistical significance is 183 observed between HA-UPy incubated with hyaluronidase and controls (i.e., HA and HA-UPy in 184 the absence of hyaluronidase) (Fig. 3e). The diminished degradation of HA-UPy is likely due to 185 the UPy-mediated network formation, which could shield the enzyme-specific binding sites from 186 hyaluronidase. ${ }^{[21]}$ 
188 Given the direct contact between HA-UPy and the cartilage surface, we also evaluated the 189 cytocompatibility of HA-UPy by exposing rat cartilage explants to HA-UPy for a duration of 7 190 days. The live/dead analyses showed that nearly $100 \%$ of the chondrocytes were alive with no 191 detrimental effect (Fig. 3f).

\section{Self-healing improved the in vivo retention of $\mathrm{HA}$}

194 The in vivo retention of HA-UPy following intraarticular injection was studied as a function of 195 time by live imaging of rat knees and compared against corresponding HA. Cy7-conjugated HA196 UPy and HA molecules were injected into rat knees and monitored using IVIS for 28 days. We

197 performed calibration studies to ensure that both cohorts received similar levels of Cy7 molecules.

198 The animals were imaged immediately and $24 \mathrm{~h}$ post-injection for the initial reading, which 199 showed clear positive signals from the joints administered with the HA-UPy and HA molecules. 200 Longitudinal imaging indicated that a majority $(>60 \%)$ of the HA was cleared from the joint by $201 \sim 3 \mathrm{~d}$ (Figs. 4a, b). In contrast, strong positive signals were present in the HA-UPy group even at $20228 \mathrm{~d}$, the maximum experimental time point, with a $40 \%$ reduction in fluorescence intensity 203 compared to the initial reading (i.e., immediately after administration) (Fig. 4b). The values are 204 presented as a percentage of initial fluorescence intensity to account for variability among the 205 animals.

207 The effect of injury on lubricant clearance was examined by comparing the retention of HA-UPy 208 in rat joints which underwent minimally invasive anterior cruciate ligament transection (mi209 ACLT), where the HA-UPy molecules were administered two days post-mi-ACLT. Similar to the 


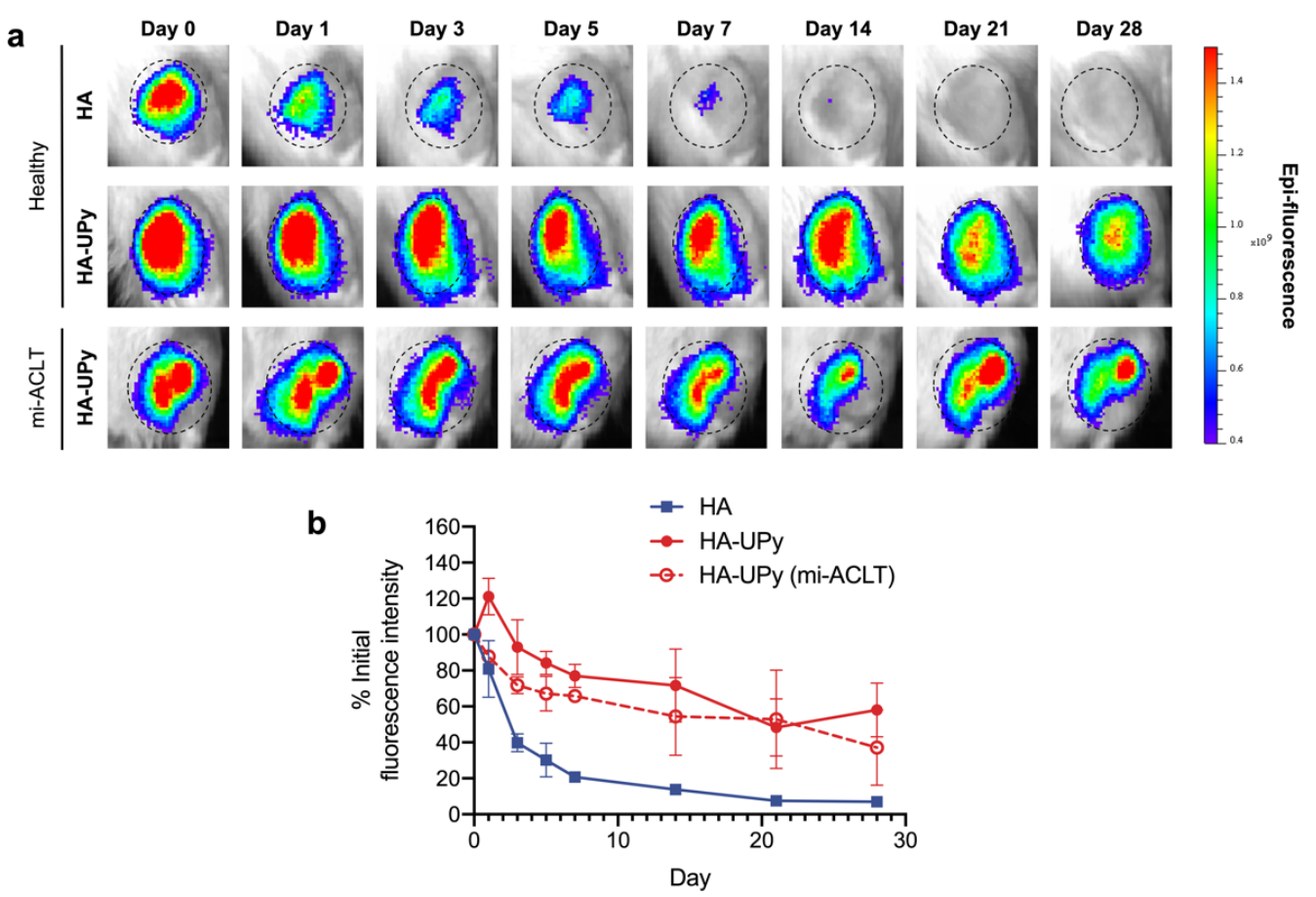

Figure 4. In vivo retention of self-healing HA. $\boldsymbol{a}$, Representative IVIS images of rat knee joints following intra-articular injection of Cy7-tagged HA or HA-UPy as a function of time. Dashed circles demarcate the joint region of interest (ROI) that was used for fluorescence intensity quantification. Color map reflects the epi-fluorescence intensity with red being the strongest. $\boldsymbol{b}$, Quantification of fluorescence intensity of ROI as a percentage of initial intensity. HA: n=2, HA-UPy: n=3, HA-UPy (mi-ACLT): $n=2$. Data are presented as means ( \pm s.e.m.).

211 healthy group, a significant amount of the administered HA-UPy was retained within the mi-ACLT

212 joints, albeit less than that in the uninjured joints. Given that a molecular weight of $200 \mathrm{kDa}$ is

213 well below the permeability barrier of the synovial membrane, ${ }^{[2]}$ we attribute the increased

214 residence time of HA-UPy within the joint synovial space to self-healing of HA molecules. 
217 The enhanced lubrication along with its improved retention in the joint suggests that the self-

218 healing HA-UPy could offer chondroprotection following joint injury. To assess the in vivo 219 chondroprotective function, we have used mouse and rat ACL transection models. The surgical

220 ACL transection model is widely used to represent articular cartilage degeneration consistent with

221 ACL injuries, which cause joint instability, chronic inflammation, and degeneration. ${ }^{[23]}$ The ACL-

222 transected mice received weekly intraarticular injections of HA-UPy, HA, or saline for four weeks

223 beginning one week post-surgery as shown in the experimental timeline (Supplementary Fig.

224 11a). Weekly injections were chosen based on prior reports ${ }^{[24]}$ and in vivo imaging which showed 225 complete clearance of HA by day 7. Safranin O staining of the knee joints at week 5 showed 226 significant damage to the articular surfaces of the cohorts that received saline (Supplementary

227 Fig. 11b). Similar to the saline group, the animals that received HA injections showed significant 228 cartilage degeneration. In contrast, the cohort that received HA-UPy maintained better cartilage 229 integrity with significant positive staining for glycosaminoglycans. We used a semi-quantitative 230 score of cartilage degeneration (OARSI) to assess the matrix loss, surface fibrillation, and erosion 231 in the joint, which corroborated the histological findings (Supplementary Fig. 11c). The lower 232 scoring value for the animals that received HA-UPy suggests improved chondroprotective function 233 of the parent HA following modification (i.e., HA-UPy).

235 Because mouse joints only permit intraarticular injections of small volumes $(\sim 5 \mu \mathrm{L})$, we employed 236 a rat knee injury model to determine the chondroprotection of self-healing HA-UPy. By using a 237 minimally invasive, percutaneous procedure, we have developed an ACL injury (mi-ACLT) in the 238 rat knee without surgically opening the joint. Specifically, the ACL was transected with an 18G 239 needle which was inserted into the knee joint lateral to the patellar ligament while the knee was 
240 flexed at a $\sim 120^{\circ}$ angle (Supplementary Fig. 12a). Successful ACL rupture was confirmed by

241 using the anterior drawer test, which exhibited abnormal subluxation of the tibia. The dissected

242 knee joints post-mortem showed that the ACL had been successfully transected (Supplementary

243 Fig. 12b). The mi-ACLT-mediated cartilage degeneration was assessed at week 9 following

244 weekly saline injections over eight weeks. Safranin O/Fast Green staining of sagittal sections of

245 the articular joints showed severe fibrillation and erosion of both the tibial and femoral cartilage

246 (Supplementary Fig. 12c). The formation of osteophytes was also visible on the posterior region

247 of the tibia. In contrast, the cartilage surfaces of the uninjured contralateral limbs were smooth

248 with no significant degeneration, and no osteophytes were present. The degree of degeneration

249 was quantified using the rat OARSI score, ${ }^{[25]}$ which showed that the mi-ACLT group had a 250 significantly higher score than that of the unoperated control, consistent with greater cartilage

251 degeneration (Supplementary Fig. 12d).

253 The extent of cartilage degeneration was further examined by immunohistochemical (IHC)

254 staining for catabolic markers - matrix metalloproteinase-13 (MMP-13) and a disintegrin and

255 metalloproteinase with thrombospondin motifs 5 (ADAMTS-5), which are shown to be highly

256 active during cartilage degeneration. ${ }^{[26]}$ Cartilage in the mi-ACLT group showed a higher

257 expression of both ADAMTS-5 and MMP-13 than the contralateral group, indicating greater

258 degeneration (Supplementary Fig. 12e). Furthermore, clustering of the chondrocytes, a hallmark

259 of osteoarthritic cartilage, ${ }^{[27]}$ was clearly present in the mi-ACLT group but not in the healthy

260 contralateral group. In addition to the semi-quantitative scoring (OARSI score), total cartilage

261 degeneration (matrix, proteoglycan, or chondrocyte loss), significant cartilage degeneration

262 (degeneration $>50 \%$ of cartilage thickness), surface matrix loss (matrix fibrillation), and the depth 
263 ratio of cartilage lesions (ratio of depth of cartilage degeneration to total cartilage thickness,

264 measured at three zones) were quantified as described elsewhere. ${ }^{[25]}$ In addition to the tibia (which

265 is commonly the focus for rat OARSI scoring), the femur was also analyzed, as recent studies have

266 shown that the medial femoral condyle exhibits severe degeneration with ACL injury, both in

267 animals $^{[28]}$ and humans. ${ }^{[29]}$ The mi-ACLT was shown to significantly increase total degeneration

268 (Supplementary Fig. 12f), significant cartilage degeneration (Supplementary Fig. 12g), surface

269 matrix loss (Supplementary Fig. 12h), and depth ratio of cartilage lesions (Supplementary Fig.

270 12i) as compared to the healthy contralateral group. Together the data demonstrate significant

271 cartilage degeneration following mi-ACLT.

272

273 We next examined the chondroprotective function of self-healing HA in the rat mi-ACLT model.

274 Because the HA- and saline-treated animals exhibited similar cartilage degeneration, we have

275 compared the HA-UPy-treated rat joints to those treated with corresponding HA. As described in

276 Fig. 5a, the animals received weekly injections starting one day post-mi-ACLT for a total of eight

277 weeks. At week 9, animals were euthanized, and their joints were examined histologically. The

278 cohorts treated with HA showed significant cartilage degeneration compared to those treated with

279 HA-UPy. Specifically, cartilage erosion, proteoglycan loss, and osteophytes in the tibia were

280 clearly observed in the HA group and were similar to features observed in the saline group (Fig.

281 5b). On the contrary, joints treated with HA-UPy showed higher Safranin O staining intensity with

282 minimal cartilage thinning but displayed some degree of cartilage fibrillation and osteophyte

283 formation. In comparing the OARSI scores, HA-UPy, while higher than the contralateral group,

284 had a significantly lower score than the corresponding HA group (Fig. 5c). 
a

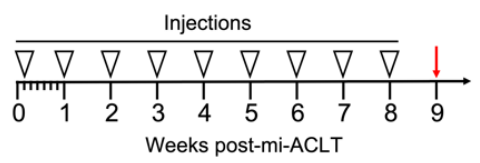

b
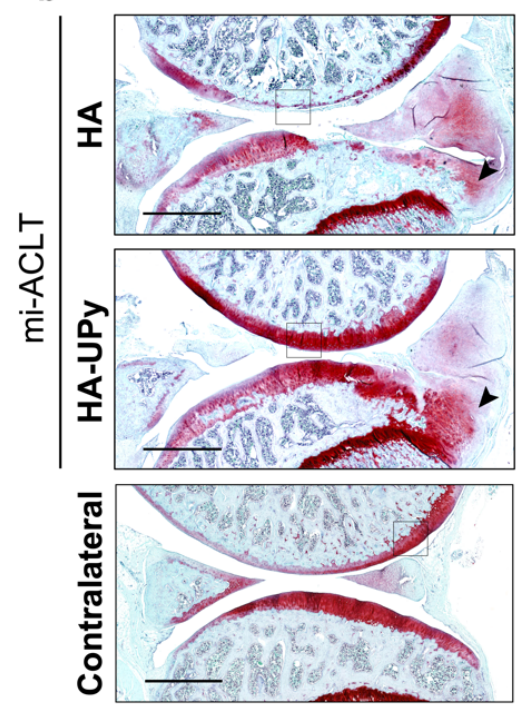
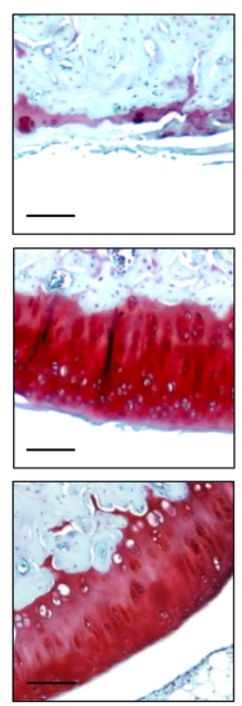

c

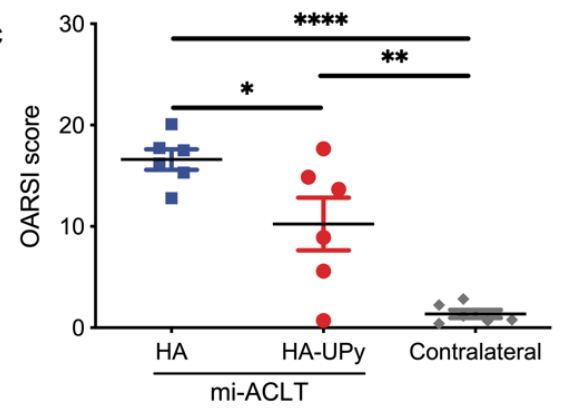

d
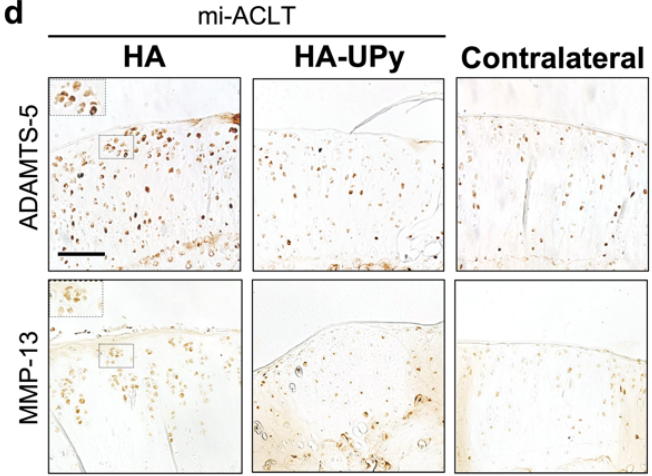

e

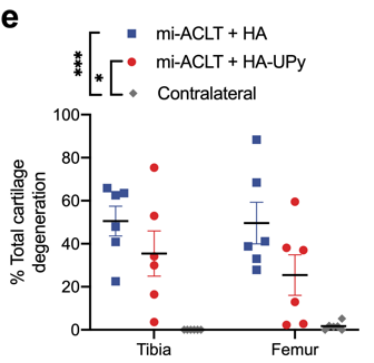

f

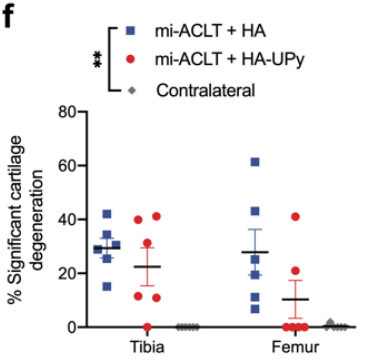

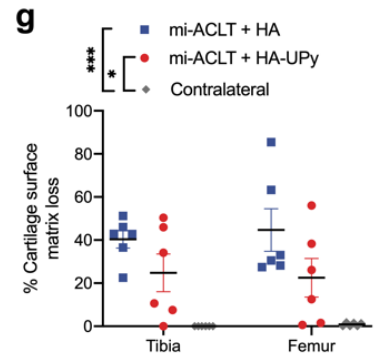

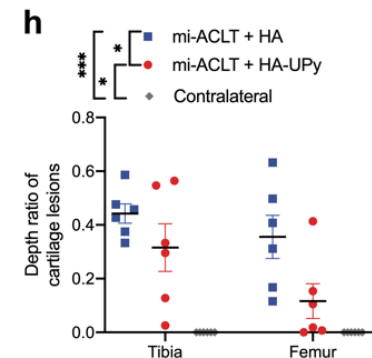

Figure 5. Chondroprotection of self-healing HA in a minimally invasive rat ACLT model. $\boldsymbol{a}$, Experimental timeline showing schedule of injections. $\boldsymbol{b}$, Safranin O-stained mi-ACLT joint treated with HA showed severe cartilage degeneration with an osteophyte in the tibia (arrow). HA-UPy-injected joints showed strong proteoglycan staining while exhibiting some cartilage fibrillation and osteophyte formation (arrow). Contralateral joints without injury were used as a positive control. Scale bar: $1 \mathrm{~mm}$. c, OARSI scoring indicates that the HA-UPy group had significantly less degeneration than the unmodified HA group. Data are presented as means $( \pm$ s.e.m.) and statistical significance was analyzed using one-way ANOVA with Tukey's multiple comparisons test. $\boldsymbol{d}$, ADAMTS-5 and MMP-13 IHC staining of tibial cartilage. Greater positive staining and chondrocyte clustering (inset) is observed in the HA group compared to the HAUPy group. Scale bar: $100 \mu \mathrm{m}$. $\boldsymbol{e}$ - $\boldsymbol{h}$, Quantitative measures of (e) total cartilage degeneration, (f) significant cartilage degeneration, $(\boldsymbol{g})$ cartilage surface matrix loss, and (h) thickness of cartilage lesions for both the tibia and femur. Data are presented as means ( \pm s.e.m.). A twoway repeated measures ANOVA was used to analyze statistical significance with Tukey's multiple comparisons used to analyze the differences between treatments. The significance in the legend shows the Tukey's multiple comparisons between treatments. Significance is determined as $* P<0.05$, $* * P<0.01$, and $* * * P<0.001$. 
Furthermore, MMP-13 and ADAMTS-5 IHC staining showed higher expression of these catabolic enzymes in the HA group, as seen by greater staining (both in intensity and the number of stained cells), compared to the HA-UPy and contralateral groups (Fig. 5d). Furthermore, the joints treated with HA showed evidence of chondrocyte clustering, similar to those treated with saline. A majority of the HA-UPy-treated joints showed minimal cartilage degeneration, and no chondrocyte clustering was observed in these animals similar to the unoperated contralateral groups. Moreover, the organization and distribution of chondrocytes within the cartilage of cohorts treated with the HA-UPy molecules was found to be similar to that of the uninjured contralateral groups. We also quantitively assessed the total cartilage degeneration, significant cartilage degeneration, surface matrix loss, and the depth ratio of cartilage lesions. These parameters were lower in the HA-UPy group than the HA group for both the femur and tibia (Figs. 5e-h). Despite the high variability among the treated animals, the total tibial degeneration was $\sim 30 \%$ less in animals treated with HAUPy compared to those treated with HA (as a percentage of total cartilage width: $35 \pm 10 \%$ for HAUPy vs. $51 \pm 7 \%$ for HA). Similarly, joints treated with HA-UPy displayed half as much total cartilage degeneration in the femur (as a percentage of total cartilage width: $25 \pm 9 \%$ for HA-UPy vs. $50 \pm 10 \%$ for HA) compared to those treated with HA. The HA-UPy group also showed a reduced amount of significant cartilage degeneration, which consists of the width of cartilage in which $50 \%$ or more of the cartilage thickness is degenerated. The cartilage lesions in the HA-UPy group also spanned significantly less of the cartilage thickness as compared to those in the HA group were also significantly less deep than the HA group, indicating that self-healing HA was 307 more chondroprotective than unmodified HA. The high variability observed in the HA-UPytreated group could be attributed to the presence of minimally modified or unmodified HA molecules. It is also likely that the high variability is due to differences in the initial cartilage 
310 damage that may result from the needle during the mi-ACLT procedure. Because the injury is

311 performed on a closed knee, there is risk of unintentionally damaging the cartilage or other joint

312 tissues, increasing the severity of the injury. While the potential for this variability is high, we have

313 randomized the animals to each treatment to ensure that differences due to the mi-ACLT procedure

314 are spread amongst groups.

\section{Conclusion}

317 Taken together, our results show that endowing HA with self-healing functionality can be used to

318 improve the intraarticular longevity of HA. Introduction of self-healing moieties not only

319 improved the residence time and lubrication properties of HA, but also endowed the precursors

320 with biological functions otherwise lacking. In particular, self-healing HA showed improved

321 viscoelastic properties without compromising its injectability. These modified HA molecules also

322 exhibited enhanced free radical scavenging and diminished enzymatic degradation. Moreover,

323 intraarticular administration of self-healing HA can be used to slow the progression of cartilage

324 degeneration after trauma. In this proof-of-concept study, we used low molecular weight (200

$325 \mathrm{kDa}) \mathrm{HA}$ as the precursor, which was necessary to limit the confounding contributions arising

326 from high molecular weight HA. Having demonstrated the promising beneficial effect of self-

327 healing HA, future studies will include self-healing HA generated from high molecular weight HA

328 precursors in a larger animal model. Our approach of using molecular engineering to imbue

329 biomaterials with self-healing could serve as a guide for engineering self-regenerating biomaterials

330 and lubricants with greater efficacy and therapeutic outcome. 
Methods

334 Synthesis of UPy-bearing linker: UPy-bearing linkers were synthesized via a multi-step 335 process. ${ }^{[11 \mathrm{a}, 30]}$ Briefly, 2-amino-4-hydroxy-6-methylpyrimidine (Sigma, Cat.\# A58003) (10 g, $3360.08 \mathrm{~mol}$ ) was dissolved in excess 1,6-diisocyanatohexane (Sigma, Cat.\# 52650) (107 g, 0.64 mol) 337 and reacted at $100^{\circ} \mathrm{C}$ overnight in argon environment. The product, termed as compound $1-1$ 338 (6-isocyanatohexyl)-3-(6-methyl-4-oxo-1,4-dihydropyrimidin-2-yl) urea in Fig. 1b, was 339 precipitated in n-pentane, filtered, and dried. The product was characterized by using NMR and 340 FTIR (Supplementary Figs. 1 and 5). Compound 1 (5 g, 0.017 mol) was mixed with $N$-boc-1,6341 hexanediamine (TCI Chemicals, Cat.\# A1375) (5.5 g, $0.025 \mathrm{~mol})$ in anhydrous dichloromethane $342(\sim 75 \mathrm{~mL})$ and kept at $50^{\circ} \mathrm{C}$ overnight to yield compound 2 (tert-butyl(6-(3-(6-(3-(6-methyl-4-oxo343 1,4-dihydropyrimidin-2-yl)ureido)hexyl) ureido)hexyl)carbamate), which was precipitated in 344 chilled diethyl ether, filtered, and dried. NMR and FTIR spectra are provided in supplementary 345 information (Supplementary Figs. 2 and 5). Compound 2 (5 g) was dispersed in dichloromethane $346(90 \mathrm{~mL})$. Trifluoroacetic acid (TCI Chemicals, Cat.\# A12198) (10 mL) was added to the 347 suspension and stirred vigorously at room temperature for $\sim 6 \mathrm{~h}$. Following the reaction, the 348 dichloromethane and trifluoroacetic acid were removed using a rotavapor. The solid residue was 349 dissolved in minimum amount of dichloromethane and precipitated in excess chilled acetone. The 350 product, compound 3, (1-(6-(3-(6-aminohexyl)ureido)hexyl)-3-(6-methyl-4-oxo-1,4351 dihydropyrimidin-2-yl)urea-trifluoroacetic acid) was filtered, washed repeatedly with acetone and 352 dried in vacuum. The product was characterized by NMR and FTIR analyses (Supplementary 353 Figs. 3 and 5). The dried compound 3 was treated with Amberlite IRA 400 chloride ion exchange 354 resin (Sigma, Cat.\# 247669) in dimethylsulfoxide-water mixture (1:1) at room temperature for 2 355 h. The resin was filtered off and the solution of the UPy-bearing linker (compound 4, 1-(6-(3-(6- 
aminohexyl)ureido)hexyl)-3-(6-methyl-4-oxo-1,4-dihydropyrimidin -2-yl)urea $\mathrm{HCl}$ ) was used to react with HA. The product was characterized by NMR and FTIR spectra (Supplementary Figs. 4 and 5).

Synthesis of HA-UPy: To synthesize HA-UPy, UPy-bearing linker was reacted with sodium hyaluronate (HA, MW 200 kDa or 1MDa; Lifecore Biomedical, Cat\# HA200K) using EDC/NHS chemistry. Briefly, HA was dissolved in a mixture of deionized water and DMSO $(1: 1)$ at 5 $\mathrm{mg} / \mathrm{mL}$, to which 1-ethyl-3-(3-dimethylaminopropyl)carbodiimide hydrochloride (EDC, TCI Chemicals, Cat.\# D1601), N-hydroxysuccinimide (NHS, Sigma, Cat.\# 130672), and compound 4

365 (each 1 equivalent with respect to the carboxylic acid groups of HA) were sequentially added at 36615 min intervals. The reaction was carried out at room temperature for $48 \mathrm{~h}$, and the resulting HA367 UPy product was purified via dialysis against water and lyophilized. Successful conjugation of 368 UPy to HA was verified by a combination of FTIR and NMR spectroscopy, and the extent of UPy conjugation was quantified via ${ }^{1} \mathrm{HNMR}$ spectroscopy.

371 Synthesis of HA-Cy 7 and HA-UPy-Cy7: Cyanine 7 (Cy7, Lumiprobe, Cat.\# 550C0) dye was

372 conjugated onto HA and HA-UPy via amide coupling reaction. Briefly, HA or HA-UPy was

373 dissolved in a mixture of deionized water and DMSO (1:1) at $5 \mathrm{mg} / \mathrm{mL}$. EDC (1 equivalent with

374 respect to the carboxylic acid group of HA or HA-UPy), NHS (1 equivalent with respect to the

375 carboxylic acid group of HA or HA-UPy), and $\mathrm{Cy} 7$ (0.04 equivalent with respect to the carboxylic 376 acid group of HA or HA-UPy) were subsequently added to the polymer solution. After $48 \mathrm{~h}$ of 377 reaction at room temperature, the mixture was dialyzed extensively with water for $4 \mathrm{~d}$. The 378 solutions were then freeze-dried to obtain HA-Cy7 or HA-UPy-Cy7. The product was 
characterized by a combination of FTIR and NMR spectroscopy, and the extent of dye conjugation was quantified via $\mathrm{UV} /$ vis absorption spectroscopy.

\section{Fourier transform infrared (FTIR) and nuclear magnetic resonance ( ${ }^{1}$ HNMR) spectroscopy:} FTIR spectrometer (Nicolet 8700) with an attenuated total reflection (ATR) range of 4000 to 650 $\mathrm{cm}^{-1}$ was used for all characterization. For ${ }^{1} \mathrm{HNMR}$ measurements, the products were dissolved in heavy water $(\sim 1 \mathrm{wt} \%)$, and the spectra were recorded by using a $500 \mathrm{MHz}$ Agilent/Varian VNMRS spectrometer at either room temperature or $80^{\circ} \mathrm{C}$.

Gelation of HA and HA-UPy molecules: HA and HA-UPy solutions of various concentrations (2 $\mathrm{wt} \%, 5 \mathrm{wt} \%$, and $10 \mathrm{wt} \%$ ) were prepared by dissolving the required weight of the molecules in phosphate-buffered saline (PBS). For visualizing gelation, food dye was added. Eppendorf tubes containing the solutions were inverted to visualize the flow under gravity, and images were taken both immediately following dissolution and after $24 \mathrm{~h}$.

Rheological analysis: Both HA-UPy and HA were prepared in PBS and subjected to rheological measurements as a function of concentration by using a rotational rheometer (AR-G2, TA Instruments). Each sample was loaded on a parallel plate geometry (Al, diameter $8 \mathrm{~mm}$, and the oscillatory frequency sweep measurements were conducted at $1 \%$ strain amplitude with frequencies ranging from 0.1 to $10 \mathrm{~Hz}$. To assess the shear-thinning behavior, the steady-state viscosities of HA-UPy and HA at $10 \mathrm{wt} \%$ were measured at $1 \%$ strain as a function of shear rate ( 0.1 to $\left.100 \mathrm{~s}^{-1}\right)$. To evaluate the recovery of HA-UPy and HA at $10 \mathrm{wt} \%$, step-strain measurements were recorded at $1 \mathrm{rad} / \mathrm{s}$ with a range of consecutive strains $(1 \%, 100 \%, 1 \%, 1000 \%$, and $1 \%)$ 
402

403

404

405

406

407

408

409

410

411

412

413

414

415

416

417

418

419

420

421

422

423 Free radical scavenging: The ability of HA-UPy to scavenge free radicals was analyzed by using

424 1,1-diphenyl-2-picrylhydrazyl (DPPH; Sigma, Cat.\# D9132) or Fenton reagent as free radical

applied each for $180 \mathrm{~s}$. To examine hysteresis of HA-UPy, 6 cycles of alternative low (1\%) and high (500\%) strain were applied. All samples were measured in triplicate.

Injectability of HA-UPy molecules: $10 \mathrm{wt} \%$ HA-UPy molecules were generated in PBS, loaded into a Hamilton syringe, and extruded into different shapes through a $26 \mathrm{G}$ needle.

Self-healing of HA-UPy molecules: To examine the self-healing phenomenon, hydrogel pieces were generated from HA-UPy (10 wt $\%$ and colored differently for visualization). Several pieces of the hydrogel were gently brought into contact with one another.

Enzymatic degradation: The stability of HA-UPy was evaluated in the presence of hyaluronidase (Sigma, Cat.\# H3506). ${ }^{[21]}$ In brief, HA-UPy and HA were dissolved in $20 \mathrm{mM}$ sodium acetate buffered solution (pH 6) at $2.5 \mathrm{mg} / \mathrm{mL}$ supplemented with $1 \mathrm{kU} / \mathrm{mL}$ hyaluronidase. Each sample was sealed in a benzoylated dialysis membrane (MWCO $\sim 2 \mathrm{kDa}$; Sigma, Cat.\# D2272) and dialyzed against sodium acetate buffer at $37^{\circ} \mathrm{C}$ for $48 \mathrm{~h}$. The dialysate containing the degradation products was collected for uronic acid assay. The solution was mixed with $12.5 \mathrm{mM}$ sodium tetraborate (Alfa Aesar, Cat.\# A16176) in concentrated sulfuric acid at a volume ratio of 1:6 and heated at $100^{\circ} \mathrm{C}$ for 10 mins. Upon cooling, $0.15 \%$ m-hydroxydiphenyl (Sigma, Cat.\# 262250) in $0.5 \% \mathrm{NaOH}$ was added and its absorbance was measured at $520 \mathrm{~nm}$ using UV/vis spectroscopy. Known concentrations of HA $(0-2.5 \mathrm{mg} / \mathrm{mL})$ were used to generate the standard curve. 
425 sources. ${ }^{[16]}$ For the DPPH assay, $10 \mathrm{wt} \%$ HA-UPy or HA was fully soaked in ethanol containing

$4260.1 \mathrm{mM} \mathrm{DPPH}$ at $37^{\circ} \mathrm{C}$ for $1 \mathrm{~h}$ in the dark. Saline of the same volume was used as the control. The

427 absorbance of DPPH solution at $517 \mathrm{~nm}$ before $\left(\mathrm{Abs}_{0}\right)$ and after $\left(\mathrm{Abs}_{\mathrm{t}}\right)$ the incubation was recorded

428 using UV/vis. The DPPH scavenging effect was determined as $\frac{A b s_{0}-A b s_{t}}{A b s_{0}} \times 100 \%$. The average

429 reduction in absorbance in the saline group was subtracted from the HA and HA-UPy groups to

430 normalize for dilution. All samples were measured in triplicate.

431

432 To examine the hydroxyl radical scavenging effect, the Fenton reagent was prepared as described

433 elsewhere. ${ }^{[31]}$ Briefly, a reaction mixture consisting of $1 \mathrm{mM}$ ferric chloride (Sigma, Cat.\#

434 157740), 30 mM deoxyribose (Sigma, Cat.\# 121649), 1 mM ascorbic acid (Sigma, Cat.\# A92902),

$4351 \mathrm{mM}$ EDTA (Sigma, Cat.\# E9884) and $20 \mathrm{mM} \mathrm{H}_{2} \mathrm{O}_{2}$ was prepared in $0.2 \mathrm{M}$ phosphate buffer. 1

$436 \mathrm{~mL}$ of the reaction mixture was added to either $100 \mu \mathrm{L}$ of $10 \mathrm{wt} \%$ HA-UPy or $0.2 \mathrm{M}$ phosphate

437 buffer (control). The gel or phosphate buffer were incubated at room temperature for $1 \mathrm{~h}$ on a

438 shaker plate. Following the incubation period, $500 \mu \mathrm{L}$ of the reaction mixture was taken from each

439 tube and mixed with $500 \mu \mathrm{L}$ of a solution of $0.25 \%$ thiobarbituric acid (TBA; Sigma, Cat.\# T5500)

440 in 15\% trichloroacetic acid (TCA; Sigma, Cat.\# T6399). The mixtures were incubated in a silicon

441 oil bath at $100^{\circ} \mathrm{C}$ for $20 \mathrm{~min}$. The absorbance was measured at $532 \mathrm{~nm}$ using a UV/Vis

442 spectrophotometer, with a lower absorbance corresponding to fewer hydroxyl radicals. All samples

443 were measured in triplicate.

444

445 Following exposure to the Fenton reaction mixture, the samples were washed in PBS, freeze dried, 446 and reconstituted in saline at a concentration of $10 \mathrm{wt} \%$. Corresponding HA-UPy samples were 447 similarly incubated in phosphate buffer alone (controls), followed by washing in PBS, freeze 
448 drying, and reconstituting in saline. A frequency sweep was performed as previously described at

$4491 \%$ strain under oscillatory mode with frequency varying from 0.1 to $10 \mathrm{~Hz}$. The storage moduli

$450\left(\mathrm{G}^{\prime}\right)$ at $1 \mathrm{~Hz}$ was compared for the free radical-treated HA-UPy and corresponding HA-UPy

451 control.

452

453 Explant coculture: To examine the biocompatibility of HA-UPy, rat tibial condyles were

454 harvested and cultured in chondrocyte medium with or without HA-UPy $(10 \mathrm{wt} \%, 50 \mu \mathrm{L})$ for $7 \mathrm{~d}$.

455 The cartilage explants were then rinsed and incubated in PBS containing $0.05 \%$ calcein

456 acetoxymethyl and $0.2 \%$ ethidium homodimer-1 from the Live/Dead Cell Viability Assays kit

457 (Life technologies, Cat.\# L3224) for $30 \mathrm{~min}$. After thorough washing, the explants were sectioned 458 and imaged using a Keyence (BZ-X710) microscope.

460 Coefficient of friction between cartilage explants: Two flat cartilage discs (8-mm diameter and

$461 \quad 0.5$-mm thickness, porcine, 3-year-old) were mounted on sandpaper-covered parallel plates using 462 cyanoacrylate glue, with the articular surfaces facing each other. After equilibration in saline, HA, 463 HA-UPy $(10 \mathrm{wt} \%)$, or saline was introduced at the cartilage-cartilage interface, the discs were

464 brought into contact and programmed to move against each other by using a rotational rheometer.

465 Both normal stress and shear stress were recorded under shear rates ranging from 0.1 to $1 \mathrm{~s}^{-1}$. The

466 coefficient of friction $(\mu)$ at the cartilage-cartilage interface was calculated using the equation, $\mu=$

$467 \quad \frac{\text { Shear stress }}{\text { Normal stress }}$.

469 ACL injury models: All animal studies were approved by the Institutional Animal Care and Use 470 Committee at Duke University in compliance with NIH guidelines for laboratory animal care. Both 
471 female mice (C57BL/6J, 3-month-old, Jackson Lab) and rats (Lewis, 3-month-old, Charles River)

472 were used for unilateral anterior cruciate ligament transection (ACLT). Each animal was sedated

473 using $2 \%$ isoflurane and injected with buprenorphine ( $1 \mathrm{mg} / \mathrm{kg}$, sustained release, ZooPharm) as

474 an analgesic prior to surgery.

475 For ACLT in mouse, each animal was placed in a supine position with the left hindlimb bent over

476 a triangular cradle. After shaving and disinfecting the skin, a cut less than 0.5 -cm-long was created

477 from the medial side to expose the joint capsule. The ACL was fully extended by bending the knee

478 to $90^{\circ} \mathrm{C}$ and transected using spring scissors (FST, Cat.\# 15004-08). Bupivacaine (0.5\%, Hospira)

479 was then applied topically, and the incision was closed with Vicryl 5-0 sutures.

480 For mi-ACLT in rat, the left hindlimb was disinfected and flexed to approximately a $90^{\circ}$ angle.

481 An 18G needle was inserted perpendicularly into the joint on the lateral side of the patellar

482 ligament, and the bevel of the needle was used to transect the ACL. To confirm the completion of

483 ACLT, the clinical anterior drawer test was performed. In the anterior drawer test, the tibia easily

484 moved out of the normal range of motion when pressure was applied behind the tibia while the

485 femur was held in place.

486 Following surgery, animals were placed on heating pads to aid in recovery from anesthesia and

487 left unconstrained in cages.

489 Intra-articular injections: Sterile saline, HA (200 kDa, $10 \mathrm{wt} \%$ in saline), and HA-UPy (200 kDa,

$49010 \mathrm{wt} \%$ in saline) were prepared fresh and injected into the injured joints using a Hamilton syringe

491 fitted with $26 \mathrm{G}$ needle. Animals were randomized to treatment groups following surgery. For

492 mouse, injections were started one week following ACLT and performed weekly for four weeks 
493 with $5 \mu \mathrm{L}$ of sample injected each time. For rat, injections were started one day following mi-

494 ACLT and repeated weekly for another eight weeks with $50 \mu \mathrm{L}$ of sample injected each time. One 495 week after the last injection, animals were euthanized.

497 IVIS imaging: Calibration studies with varying extent of Cy7 modified HA molecules were 498 carried out to identify optimal Cy7 concentration required to obtain the optical intensity. $50 \mu \mathrm{L}$ of 499 Cy7-containing HA or HA-UPy $(10 \mathrm{wt} \%)$ was administered into the rat joint via intra-articular 500 injection. At designated times after injection, rats were anesthetized under isoflurane inhalation 501 and imaged by using an IVIS Kinetics system (excitation filter, $745 \mathrm{~nm}$; emission filter, ICG; 502 excitation time, $100 \mathrm{~ms}$ ). The epi-fluorescence intensity of $\mathrm{Cy} 7$ in the joint was quantified by 503 selecting an ROI from images taken at Day 0 . The percent of initial fluorescence intensity was 504 calculated after measuring the fluorescence intensity at each subsequent time point.

506 Histological analysis: Fixed joint samples were decalcified in 14\% EDTA (pH 8.0, Sigma, Cat.\# 507 E5134) for 2-3 weeks, rinsed with PBS, dehydrated, and embedded in paraffin blocks, and sliced 508 into $8 \mu \mathrm{m}$-thick sections using a Leica rotary microtome. Each section was deparaffinized in 509 CitriSolv (Decon Labs, Cat.\# 1601) and rehydrated through graded alcohols and deionized water. 510 For Safranin-O staining, the rehydrated sections were incubated in 1\% Safranin-O (Sigma, Cat.\# 511 S8884) for $30 \mathrm{~min}$ for mouse cartilage sections and $1 \mathrm{~h}$ for rat cartilage sections. They were 512 counter-stained with $0.02 \%$ Fast Green (Sigma, Cat.\# F7258) for 1 min for mouse and 1.5 min for 513 rat, followed by incubating in hematoxylin solution (Ricca Chemical, Cat.\# 3536-16) for 1 min for 514 mouse and 5 min for rat. The stained sections were rinsed, dehydrated, and covered with a 
mounting medium (Fisher Scientific, Cat.\# SP15-100). Images were taken using a Keyence microscope.

518 OARSI scoring: Scoring of Safranin O-stained sagittal sections was performed by three blinded 519 scorers in accordance with the OARSI histopathology initiative for evaluation of OA in mouse ${ }^{[32]}$ 520 and rat. ${ }^{[25]}$ Scoring criteria for the mouse included the degree of degeneration of cartilage in both 521 the tibia and femur, evaluated from 0-6: 0 means normal; 0.5 has loss of proteoglycan without 522 structural changes; 1 shows limited fibrillation on the cartilage surface; 2 presents vertical clefts;

5233 means vertical clefts or erosion covering $<25 \%$ of surface area; 4 for $25-50 \%$ area being affected;

5245 for $50-75 \%$; and 6 for $>75 \%$. Scoring criteria for the rat included total tibial cartilage 525 degeneration (0-5 for 3 zones, total 0-15), femoral cartilage degeneration (0-5), bone score (0-5), 526 and osteophyte score (0-4), with total added score from 0-29. The osteophyte score was modified

527 for sagittal joint sections based upon a histogram of osteophyte sizes for all samples in the mi528 ACLT groups. ImageJ was used to quantify total cartilage degeneration, significant cartilage 529 degeneration, and surface matrix loss (all expressed as the percentage of total cartilage width), as 530 well as depth ratio of cartilage lesions (expressed as the depth of degenerated cartilage to the 531 thickness of total cartilage). These measurements were completed for both the tibia and femur, the 532 latter of which is a modification of the original scoring which only examines the tibia. A total of 533 four sections, taken from two locations within the medial compartment of the joint, were evaluated.

534 These four scores/measurements were averaged as technical replicates for each subject.

536 Immunohistochemical analysis: Immunohistochemical staining was used to detect MMP-13 and 537 ADAMTS-5 expression in cartilage. Briefly, deparaffinized sections were subjected to heat- 
538 induced antigen retrieval in a vegetable steamer for $13 \mathrm{~min}$ and permeabilized with $0.1 \%$ Triton

$539 \mathrm{X}-100$ for $15 \mathrm{~min}$. Tissue sections were blocked with $0.1 \% \mathrm{BSA}$ and $0.26 \mathrm{M}$ glycerol in TBS for

$5401 \mathrm{~h}$, sequentially exposed to Dual Endogenous Enzyme Block (Dako, Cat.\# S2003) for 30 min,

541 and $5 \%$ and $1.5 \%$ normal goat serum for $30 \mathrm{~min}$ and $1 \mathrm{~h}$, respectively, before incubation at $4^{\circ} \mathrm{C}$

542 overnight with either anti-MMP13 (dilution 1:500, Abcam, Cat.\# ab39012) or anti-ADAMTS5

543 (dilution 1:100, Abcam, Cat.\# ab41037). VECTASTAIN ${ }^{\circledR}$ Elite ABC HRP Kit (Vector

544 Laboratories, Cat.\# PK-6100) kit and ImmPACT ${ }^{\circledR}$ DAB Peroxidase Substrate (Vector

545 Laboratories, Cat.\# SK-4105) were used to visualize the enzyme expression.

547 Statistical analysis: The means with standard error of mean $(\mathrm{n} \geq 3)$ are presented in the results.

548 All animal studies included at least 6 animals per group. All the data were subjected to two-tailed

549 Student's t-test, one-way analysis of variance (ANOVA) with post-hoc Tukey's multiple

550 comparisons test, or two-way repeated measures ANOVA with Tukey's multiple comparisons test

551 using GraphPad Prism 8. Any p-value of less than 0.05 was indicated with an asterisk and was

552 considered statistically significant. All experiments were reproduced independently.

553

554

555

556

557

558

559

560

561

562

563

564

565

566

\section{References}

[1] B. A. Hills, P I Mech Eng H 2000, 214, 83.

[2] a) R. C. Gupta, R. Lall, A. Srivastava, A. Sinha, Front Vet Sci 2019, 6, 192; b) M. K. Cowman, T. A. Schmidt, P. Raghavan, A. Stecco, F1000Res 2015, 4, 622.

[3] a) C. T. Stafford, W. Niedermeier, H. L. Holley, W. Pigman, Annals of the rheumatic diseases 1964, 23, 152; b) E. A. Balazs, D. Watson, I. F. Duff, S. Roseman, Arthritis \& Rheumatism 1967, 10, 357.

[4] a) A. Huynh, R. Priefer, Carbohydr Res 2020, 489, 107950; b) E. Maheu, F. Rannou, J. Y. Reginster, Semin Arthritis Rheum 2016, 45, S28. 
567 [5] a) A. Fakhari, C. Berkland, Acta Biomater 2013, 9, 7081; b) E. Trabucchi, S. Pallotta, M. Morini, F. Corsi, R. Franceschini, A. Casiraghi, A. Pravettoni, D. Foschi, P. Minghetti, Int J Tissue React 2002, 24, 65; c) L. W. Moreland, Arthritis Res Ther 2003, 5, 54.

[6] a) C. H. Evans, V. B. Kraus, L. A. Setton, Nature Reviews Rheumatology 2014, 10, 11; b) S. Bowman, M. E. Awad, M. W. Hamrick, M. Hunter, S. Fulzele, Clin Transl Med 2018, 7, 6. a) A. Singh, M. Corvelli, S. A. Unterman, K. A. Wepasnick, P. McDonnell, J. H. Elisseeff, Nature Materials 2014, 13, 988; b) H. J. Faust, S. D. Sommerfeld, S. Rathod, A. Rittenbach, S. R. Banerjee, B. M. Tsui, M. Pomper, M. L. Amzel, A. Singh, J. H. Elisseeff, Biomaterials 2018, 183, 93; c) F. A. Maulvi, T. G. Soni, D. O. Shah, Journal of Biomaterials Science, Polymer Edition 2015, 26, 1035; d) R. Salzillo, C. Schiraldi, L. Corsuto, A. D’Agostino, R. Filosa, M. De Rosa, A. La Gatta, Carbohydrate polymers 2016, 153, 275. K. Yoshioka, M. Katayama, T. Nishiyam
Musculoskelet Disord 2019, 20, 424.

[9] V. M. Goldberg, R. D. Coutts, Clin Orthop Relat Res 2004, DOI: 10.1097/00003086200402000-00021130.

[10] R. P. Sijbesma, F. H. Beijer, L. Brunsveld, B. J. B. Folmer, J. H. K. K. Hirschberg, R. F. M. Lange, J. K. L. Lowe, E. W. Meijer, Science 1997, 278, 1601.

[11] a) P. Y. W. Dankers, E. N. M. van Leeuwen, G. M. L. van Gemert, A. J. H. Spiering, M. C. Harmsen, L. A. Brouwer, H. M. Janssen, A. W. Bosman, M. J. A. van Luyn, E. W. Meijer, Biomaterials 2006, 27, 5490; b) P. Y. Dankers, T. M. Hermans, T. W. Baughman, Y. Kamikawa, R. E. Kieltyka, M. M. Bastings, H. M. Janssen, N. A. Sommerdijk, A. Larsen, M. J. Van Luyn, Advanced materials 2012, 24, 2703; c) Y. Wu, L. Wang, X. Zhao, S. Hou, B. Guo, P. X. Ma, Biomaterials 2016, 104, 18; d) M. J. G. Schotman, M. M. C. Peters, G. C. Krijger, I. van Adrichem, R. de Roos, J. L. M. Bemelmans, M. J. Pouderoijen, M. G. T. A. Rutten, K. Neef, S. A. J. Chamuleau, P. Y. W. Dankers, Advanced Healthcare Materials 2021, n/a, 2001987.

[12] a) F. H. Beijer, R. P. Sijbesma, H. Kooijman, A. L. Spek, E. W. Meijer, Journal of the American Chemical Society 1998, 120, 6761; b) M. Guo, L. M. Pitet, H. M. Wyss, M. Vos, P. Y. W. Dankers, E. W. Meijer, Journal of the American Chemical Society 2014, 136, 6969.

[13] D. A. Gibbs, E. W. Merrill, K. A. Smith, E. A. Balazs, Biopolymers 1968, 6, 777.

[14] J. Nilsson, A. Thorstensson, Acta Physiologica Scandinavica 1987, 129, 107.

[15] A. Phadke, C. Zhang, B. Arman, C.-C. Hsu, R. A. Mashelkar, A. K. Lele, M. J. Tauber, G. Arya, S. Varghese, Proceedings of the National Academy of Sciences 2012, 109, 4383.

[16] a) J. Qu, X. Zhao, Y. P. Liang, T. L. Zhang, P. X. Ma, B. L. Guo, Biomaterials 2018, 183, 185; 
[22] M. Wathier, B. A. Lakin, P. N. Bansal, S. S. Stoddart, B. D. Snyder, M. W. Grinstaff, Journal of the American Chemical Society 2013, 135, 4930.

[23] a) T. Hayami, M. Pickarski, Y. Zhuo, G. A. Wesolowski, G. A. Rodan, L. T. Duong, Bone 2006, 38, 234; b) S. Kamekura, K. Hoshi, T. Shimoaka, U. Chung, H. Chikuda, T. Yamada, M. Uchida, N. Ogata, A. Seichi, K. Nakamura, H. Kawaguchi, Osteoarthritis and Cartilage 2005, 13, 632.

[24] a) M. Ikeuchi, M. Izumi, K. Aso, N. Sugimura, T. Kato, T. Tani, Eur J Pain 2015, 19, 334; b) E. Teeple, K. A. Elsaid, G. D. Jay, L. Zhang, G. J. Badger, M. Akelman, T. F. Bliss, B. C. Fleming, Am J Sports Med 2011, 39, 164.

[25] N. Gerwin, A. M. Bendele, S. Glasson, C. S. Carlson, Osteoarthritis and Cartilage 2010, 18, S24.

[26] a) M. B. Goldring, M. Otero, Curr Opin Rheumatol 2011, 23, 471; b) M. Wang, E. R. Sampson, H. Jin, J. Li, Q. H. Ke, H. J. Im, D. Chen, Arthritis Res Ther 2013, 15, R5; c) S. S. Glasson, R. Askew, B. Sheppard, B. Carito, T. Blanchet, H.-L. Ma, C. R. Flannery, D. Peluso, K. Kanki, Z. Yang, M. K. Majumdar, E. A. Morris, Nature 2005, 434, 644; d) F. Echtermeyer, J. Bertrand, R. Dreier, I. Meinecke, K. Neugebauer, M. Fuerst, Y. J. Lee, Y. W. Song, C. Herzog, G. Theilmeier, T. Pap, Nat Med 2009, 15, 1072.

[27] M. K. Lotz, S. Otsuki, S. P. Grogan, R. Sah, R. Terkeltaub, D. D'Lima, Arthritis Rheum 2010, 62, 2206.

[28] T. Maerz, M. D. Newton, M. D. Kurdziel, P. Altman, K. Anderson, H. W. Matthew, K. C. Baker, Osteoarthritis Cartilage 2016, 24, 1918.

[29] E. G. Sutter, B. Liu, G. M. Utturkar, M. R. Widmyer, C. E. Spritzer, H. C. Cutcliffe, Z. A. Englander, A. P. Goode, W. E. Garrett, Jr., L. E. DeFrate, Am J Sports Med 2019, 47, 96.

[30] S. Hou, X. Wang, S. Park, X. Jin, P. X. Ma, Advanced Healthcare Materials 2015, 4, 1491.

[31] R. Komeri, F. G. Thankam, J. Muthu, Materials Science and Engineering: C 2017, 71, 100.

[32] S. S. Glasson, M. G. Chambers, W. B. Van Den Berg, C. B. Little, Osteoarthritis Cartilage 2010, 18 Suppl 3, S17.

Supplementary Information

Supplementary Figures 1-12

643 Supplementary Text

Supplementary Methods

\section{Acknowledgments}

647 We thank H Newman, S Sharma, and N Sangaj for helping the with the blinded OARSI scoring.

648 This material is based upon work partially supported by the National Science Foundation Graduate 
649 Research Fellowship Program (A Gilpin) under Grant No. DGE 1644868. This work was partially

650 supported by the Duke University and National Institute of Arthritis and Musculoskeletal and Skin

651 Diseases (NIAMS) of the National Institutes of Health (NIH) under Award Number NIH R01

652 AR063184. The content is solely the responsibility of the authors and does not necessarily

653 represent the official views of the funding agencies.

654

655 Author contributions

656 S.V conceived the initial idea. A.G., Y.Z., and S.V. designed the study and wrote the manuscript.

657 A.G., Y.Z., and J.H. designed and performed experiments and analyzed the data. J.H.R performed/

658 helped with synthesis. Y.Y and Y.Z performed the mouse studies. W.E performed/helped with the

659 rat ACL injuries. S.Z helped with the rheological measurements. All authors were involved in the

660 manuscript preparation

661

662 Competing interests

663 The authors declare no competing interests.

664

665 